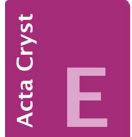
COMMUNICATIONS

ISSN 2056-9890

\title{
Crystal structure of 1-methoxy-5-methyl- $\mathrm{N}$-phenyl-1,2,3-triazole-4-carboxamide
}

\section{Inna S. Khazhieva, ${ }^{\mathrm{a} *}$ Tatiana V. Glukhareva, ${ }^{\mathrm{a}}$ Pavel A. Slepukhin ${ }^{\mathrm{b}}$ and Yury Yu. Morzherin ${ }^{\mathrm{a}}$}

${ }^{a}$ Ural Federal University, Mira 19 Ekaterinburg 620002, Russian Federation, and b. Postovsky Institute of Organic Synthesis, Kovalevskoy 22 Ekaterinburg 620090, Russian Federation. *Correspondence e-mail: i.s.khazhieva@urfu.ru

Received 17 September 2015; accepted 22 September 2015

Edited by W. T. A. Harrison, University of Aberdeen, Scotland

The title compound, $C_{11} \mathrm{H}_{12} \mathrm{~N}_{4} \mathrm{O}_{2}$, was prepared via the transformation of sodium 4-acetyl-1-phenyl-1H-[1.2.3]triazolate under the action of methoxyamine hydrochloride. The dihedral angle between the triazole and phenyl rings is $25.12(16)^{\circ}$ and the $\mathrm{C}$ atom of the methoxy group deviates from the triazole plane by 0.894 (4) $\AA$. The conformation of the CONHR-group is consolodated by an intramolecular $\mathrm{N}-$ $\mathrm{H} \cdots \mathrm{N}$ hydrogen bond to an $\mathrm{N}$-atom of the triazole ring, which closes an $S(5)$ ring. In the crystal, weak $\mathrm{N}-\mathrm{H} \cdots \mathrm{N}$ hydrogen bonds link the molecules into $C(6)[010]$ chains.

Keywords: crystal structure; 1,2,3-triazole; rearrangements; hydrogen bonding.

CCDC reference: 1426448

\section{Related literature}

For biological activities of 1.2.3-triazoles, see: Sathish Kumar \& Kavitha (2013); Khazhieva et al. (2015a). For the synthesis, see: Khazhieva et al. (2015b).

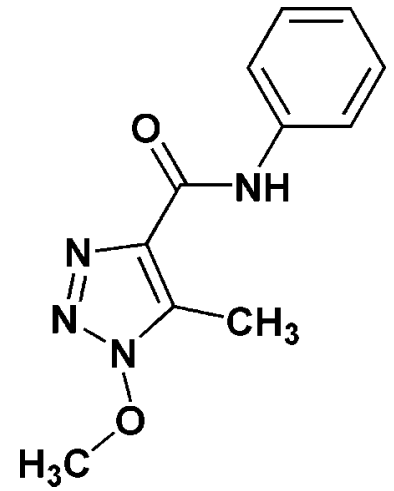

\section{Experimental}

2.1. Crystal data

$\mathrm{C}_{11} \mathrm{H}_{12} \mathrm{~N}_{4} \mathrm{O}_{2}$

$M_{r}=232.25$

Monoclinic, $P 2_{1} / c$

$a=11.4637$ (8) ̊

$b=6.4345(13) \AA$

$c=15.822(3) \AA$

$\beta=100.367(12)^{\circ}$

$V=1148.0(3) \AA^{3}$

$Z=4$

Mo $K \alpha$ radiation

$\mu=0.10 \mathrm{~mm}^{-1}$

$T=295 \mathrm{~K}$

$0.21 \times 0.16 \times 0.09 \mathrm{~mm}$

\subsection{Data collection}

Agilent Xcalibur S CCD diffractometer

7259 measured reflections

2302 independent reflections 1077 reflections with $I>2 \sigma(I)$

$R_{\text {int }}=0.040$

\subsection{Refinement}

$R\left[F^{2}>2 \sigma\left(F^{2}\right)\right]=0.055$

$w R\left(F^{2}\right)=0.147$

$S=1.00$

2302 reflections

160 parameters
$\mathrm{H}$ atoms treated by a mixture of independent and constrained refinement
$\Delta \rho_{\max }=0.43$ e $\AA^{-3}$
$\Delta \rho_{\min }=-0.22{\mathrm{e} \AA^{-3}}^{-3}$

Table 1

Hydrogen-bond geometry $\left(\AA,{ }^{\circ}\right)$.

\begin{tabular}{lllll}
\hline$D-\mathrm{H} \cdots A$ & $D-\mathrm{H}$ & $\mathrm{H} \cdots A$ & $D \cdots A$ & $D-\mathrm{H} \cdots A$ \\
\hline $\mathrm{N} 1-\mathrm{H} 1 \cdots \mathrm{N} 2$ & $0.86(2)$ & $2.33(3)$ & $2.780(4)$ & $113(2)$ \\
$\mathrm{N} 1-\mathrm{H} 1 \cdots \mathrm{N}^{\mathrm{i}}$ & $0.86(2)$ & $2.41(2)$ & $3.184(3)$ & $150(2)$ \\
\hline
\end{tabular}

Symmetry code: (i) $-x+2, y-\frac{1}{2},-z+\frac{1}{2}$.

Data collection: CrysAlis PRO (Agilent, 2006); cell refinement: CrysAlis PRO; data reduction: CrysAlis PRO; program(s) used to solve structure: SHELXS97 (Sheldrick, 2008); program(s) used to refine structure: SHELXS97 (Sheldrick, 2008); molecular graphics: publCIF (Westrip, 2010); software used to prepare material for publication: publCIF (Westrip, 2010).

\section{Acknowledgements}

We thank the Russian Foundation for Basic Research (grant 13-03-00137), State task Ministry of Education and Science of the Russian Federation No. 4.560.2014-K and the Project Enhance Competitiveness of the Ural Federal University (Project 5-100-2020)

Supporting information for this paper is available from the $\mathrm{IUCr}$ electronic archives (Reference: HB7511).

\section{References}

Agilent (2006). CrysAlis PRO. Agilent Technologies UK Ltd, Yarnton, England.

Khazhieva, I. S., Glukhareva, T. V., El'tsov, O. S., Morzherin, Yu. Yu., Minin, A. A., Pozdina, V. A. \& Ulitko, M. V. (2015b). Khim. Farm. Zh. 49, 12-15. Khazhieva, I. S., Glukhareva, T. V. \& Morzherin, Yu. Yu. (2015a). Chim. Tech. Acta, 2, 52-58.

Sathish Kumar, S. \& Kavitha, H. P. (2013). Mini-Rev. Org. Chem. 10, 40-65.

Sheldrick, G. M. (2008). Acta Cryst. A64, 112-122.

Westrip, S. P. (2010). J. Appl. Cryst. 43, 920-925. 


\section{supporting information}

Acta Cryst. (2015). E71, o798 [doi:10.1107/S2056989015017776]

\section{Crystal structure of 1-methoxy-5-methyl-N-phenyl-1,2,3-triazole-4-}

\section{carboxamide}

Inna S. Khazhieva, Tatiana V. Glukhareva, Pavel A. Slepukhin and Yury Yu. Morzherin

S1. Synthesis and crystallization

The titled compound was prepared as previously reported (Khazhieva et al., 2015b). Crystals were obtained by slow evaporation of a solution in ethanol. 


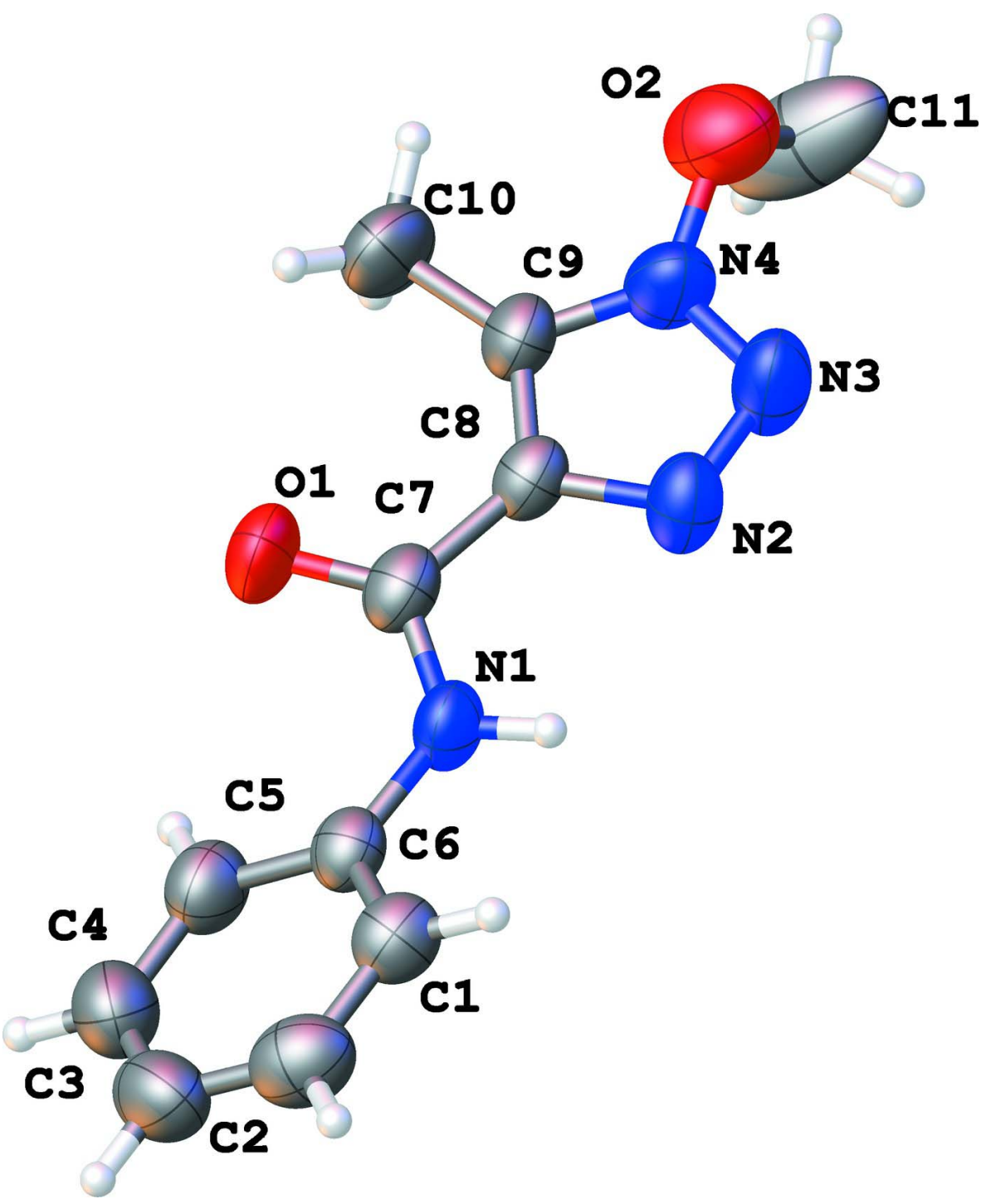

Figure 1

The molecular structure of (I), with 50\% probability displacement ellipsoids for non-H atoms.

\section{1-Methoxy-5-methyl-N-phenyl-1,2,3-triazole-4-carboxamide}

Crystal data

$\mathrm{C}_{11} \mathrm{H}_{12} \mathrm{~N}_{4} \mathrm{O}_{2}$

$M_{r}=232.25$

Monoclinic, $P 2_{1} / c$

$a=11.4637(8) \AA$

$b=6.4345(13) \AA$

$c=15.822(3) \AA$

$\beta=100.367(12)^{\circ}$

$V=1148.0(3) \AA^{3}$

$Z=4$

$D_{\mathrm{x}}=1.344 \mathrm{Mg} \mathrm{m}^{-3}$

Melting point: $310 \mathrm{~K}$

Mo $K \alpha$ radiation, $\lambda=0.71073 \AA$

Cell parameters from 1077 reflections

$\theta=2.9-26.4^{\circ}$

$\mu=0.10 \mathrm{~mm}^{-1}$

$T=295 \mathrm{~K}$

Prism, colorless

$F(000)=488$

$0.21 \times 0.16 \times 0.09 \mathrm{~mm}$ 


\section{Data collection}

Agilent Xcalibur S CCD diffractometer

Radiation source: fine-focus sealed tube Graphite monochromator $\omega$ scans

7259 measured reflections

2302 independent reflections

\section{Refinement}

Refinement on $F^{2}$

Least-squares matrix: full

$R\left[F^{2}>2 \sigma\left(F^{2}\right)\right]=0.055$

$w R\left(F^{2}\right)=0.147$

$S=1.00$

2302 reflections

160 parameters

0 restraints

Primary atom site location: structure-invariant direct methods
1077 reflections with $I>2 \sigma(I)$

$R_{\text {int }}=0.040$

$\theta_{\max }=26.4^{\circ}, \theta_{\min }=2.9^{\circ}$

$h=-7 \rightarrow 14$

$k=-5 \rightarrow 8$

$l=-19 \rightarrow 19$
Secondary atom site location: difference Fourier map

Hydrogen site location: inferred from neighbouring sites

$\mathrm{H}$ atoms treated by a mixture of independent and constrained refinement

$w=1 /\left[\sigma^{2}\left(F_{\mathrm{o}}^{2}\right)+(0.0682 P)^{2}\right]$ where $P=\left(F_{\mathrm{o}}^{2}+2 F_{\mathrm{c}}^{2}\right) / 3$

$(\Delta / \sigma)_{\max }<0.001$

$\Delta \rho_{\max }=0.43$ e $\AA^{-3}$

$\Delta \rho_{\min }=-0.22{\mathrm{e} \AA^{-3}}^{-3}$

\section{Special details}

Geometry. All e.s.d.'s (except the e.s.d. in the dihedral angle between two 1.s. planes) are estimated using the full covariance matrix. The cell e.s.d.'s are taken into account individually in the estimation of e.s.d.'s in distances, angles and torsion angles; correlations between e.s.d.'s in cell parameters are only used when they are defined by crystal symmetry. An approximate (isotropic) treatment of cell e.s.d.'s is used for estimating e.s.d.'s involving 1.s. planes.

Refinement. Refinement of $F^{2}$ against ALL reflections. The weighted $R$-factor $w R$ and goodness of fit $S$ are based on $F^{2}$, conventional $R$-factors $R$ are based on $F$, with $F$ set to zero for negative $F^{2}$. The threshold expression of $F^{2}>\sigma\left(F^{2}\right)$ is used only for calculating $R$-factors(gt) etc. and is not relevant to the choice of reflections for refinement. $R$-factors based on $F^{2}$ are statistically about twice as large as those based on $F$, and $R$-factors based on ALL data will be even larger.

Fractional atomic coordinates and isotropic or equivalent isotropic displacement parameters $\left(\AA^{2}\right)$

\begin{tabular}{lllll}
\hline & $x$ & $y$ & $z$ & $U_{\text {iso }} * / U_{\text {eq }}$ \\
\hline O1 & $0.61315(16)$ & $0.0563(3)$ & $0.15704(14)$ & $0.0777(7)$ \\
C8 & $0.7887(2)$ & $0.1712(4)$ & $0.24262(18)$ & $0.0475(7)$ \\
C6 & $0.7399(2)$ & $-0.2566(4)$ & $0.08075(18)$ & $0.0496(7)$ \\
C7 & $0.7204(2)$ & $0.0365(4)$ & $0.17728(19)$ & $0.0533(7)$ \\
N2 & $0.90562(18)$ & $0.1400(4)$ & $0.27423(17)$ & $0.0605(7)$ \\
N4 & $0.8463(2)$ & $0.3915(4)$ & $0.33811(19)$ & $0.0708(8)$ \\
C9 & $0.7489(2)$ & $0.3375(4)$ & $0.28291(19)$ & $0.0553(8)$ \\
N1 & $0.7844(2)$ & $-0.1083(4)$ & $0.14353(16)$ & $0.0530(6)$ \\
N3 & $0.9416(2)$ & $0.2771(4)$ & $0.3343(2)$ & $0.0759(8)$ \\
O2 & $0.8515(2)$ & $0.5302(4)$ & $0.40535(18)$ & $0.0956(8)$ \\
C1 & $0.7975(2)$ & $-0.4450(5)$ & $0.0824(2)$ & $0.0589(8)$ \\
H1A & 0.8634 & -0.4721 & 0.1246 & $0.071 *$ \\
C5 & $0.6434(3)$ & $-0.2172(5)$ & $0.0169(2)$ & $0.0641(8)$ \\
H5A & 0.6049 & -0.0896 & 0.0148 & $0.077 *$ \\
C3 & $0.6605(3)$ & $-0.5520(6)$ & $-0.0411(2)$ & $0.0809(10)$ \\
H3A & 0.6331 & -0.6524 & -0.0821 & $0.097 *$
\end{tabular}




\begin{tabular}{lllll} 
C2 & $0.7571(3)$ & $-0.5924(5)$ & $0.0214(2)$ & $0.0739(9)$ \\
H2A & 0.7955 & -0.7200 & 0.0225 & $0.089^{*}$ \\
C4 & $0.6048(3)$ & $-0.3651(6)$ & $-0.0430(2)$ & $0.0769(10)$ \\
H4A & 0.5395 & -0.3381 & -0.0857 & $0.092^{*}$ \\
C11 & $0.9070(4)$ & $0.7045(6)$ & $0.3901(3)$ & $0.137(2)$ \\
H11A & 0.8970 & 0.8073 & 0.4321 & $0.205^{*}$ \\
H11B & 0.8741 & 0.7551 & 0.3337 & $0.205^{*}$ \\
H11C & 0.9900 & 0.6765 & 0.3933 & $0.205^{*}$ \\
C10 & $0.6343(3)$ & $0.4512(5)$ & $0.2740(2)$ & $0.0818(10)$ \\
H10A & 0.6298 & 0.5214 & 0.3268 & $0.123^{*}$ \\
H10B & 0.5699 & 0.3543 & 0.2609 & $0.123^{*}$ \\
H10C & 0.6292 & 0.5511 & 0.2284 & $0.123^{*}$ \\
H1 & $0.858(2)$ & $-0.109(4)$ & $0.1666(17)$ & $0.048(8)^{*}$ \\
\hline
\end{tabular}

Atomic displacement parameters $\left(\AA^{2}\right)$

\begin{tabular}{lllllll}
\hline & $U^{11}$ & $U^{22}$ & $U^{33}$ & $U^{12}$ & $U^{13}$ & $U^{23}$ \\
\hline O1 & $0.0365(12)$ & $0.1021(17)$ & $0.0930(17)$ & $0.0057(10)$ & $0.0076(11)$ & $-0.0186(13)$ \\
C8 & $0.0393(15)$ & $0.0458(16)$ & $0.0589(18)$ & $0.0007(12)$ & $0.0134(13)$ & $0.0056(14)$ \\
C6 & $0.0414(15)$ & $0.0538(18)$ & $0.0549(19)$ & $-0.0035(14)$ & $0.0117(14)$ & $0.0045(16)$ \\
C7 & $0.0418(16)$ & $0.0582(18)$ & $0.062(2)$ & $-0.0016(14)$ & $0.0146(15)$ & $0.0066(16)$ \\
N2 & $0.0444(14)$ & $0.0516(15)$ & $0.0836(19)$ & $-0.0032(11)$ & $0.0062(13)$ & $-0.0019(14)$ \\
N4 & $0.0607(17)$ & $0.0658(17)$ & $0.089(2)$ & $-0.0011(13)$ & $0.0206(15)$ & $-0.0268(16)$ \\
C9 & $0.0400(16)$ & $0.065(2)$ & $0.0624(19)$ & $-0.0028(14)$ & $0.0117(15)$ & $-0.0013(16)$ \\
N1 & $0.0354(13)$ & $0.0558(15)$ & $0.0661(17)$ & $0.0045(11)$ & $0.0041(12)$ & $-0.0011(13)$ \\
N3 & $0.0465(15)$ & $0.0710(17)$ & $0.107(2)$ & $0.0004(13)$ & $0.0059(14)$ & $-0.0188(17)$ \\
O2 & $0.0967(18)$ & $0.0961(18)$ & $0.102(2)$ & $-0.0125(14)$ & $0.0395(15)$ & $-0.0152(16)$ \\
C1 & $0.0573(17)$ & $0.0563(19)$ & $0.064(2)$ & $0.0021(15)$ & $0.0140(15)$ & $0.0062(17)$ \\
C5 & $0.0512(18)$ & $0.073(2)$ & $0.067(2)$ & $0.0069(15)$ & $0.0089(16)$ & $0.0035(19)$ \\
C3 & $0.077(2)$ & $0.093(3)$ & $0.075(3)$ & $-0.018(2)$ & $0.018(2)$ & $-0.024(2)$ \\
C2 & $0.081(2)$ & $0.061(2)$ & $0.085(3)$ & $-0.0020(18)$ & $0.030(2)$ & $-0.004(2)$ \\
C4 & $0.061(2)$ & $0.098(3)$ & $0.070(2)$ & $-0.005(2)$ & $0.0055(17)$ & $-0.010(2)$ \\
C11 & $0.171(4)$ & $0.055(2)$ & $0.221(5)$ & $-0.019(2)$ & $0.133(4)$ & $-0.005(3)$ \\
C10 & $0.0566(19)$ & $0.098(2)$ & $0.092(3)$ & $0.0195(17)$ & $0.0172(17)$ & $-0.016(2)$ \\
& & & & & & \\
\hline
\end{tabular}

Geometric parameters $\left(\AA,{ }^{\circ}\right)$

\begin{tabular}{llll}
\hline $\mathrm{O} 1-\mathrm{C} 7$ & $1.220(3)$ & $\mathrm{C} 1-\mathrm{C} 2$ & $1.372(4)$ \\
$\mathrm{C} 8-\mathrm{N} 2$ & $1.359(3)$ & $\mathrm{C} 1-\mathrm{H} 1 \mathrm{~A}$ & 0.9300 \\
$\mathrm{C} 8-\mathrm{C} 9$ & $1.365(3)$ & $\mathrm{C} 5-\mathrm{C} 4$ & $1.359(4)$ \\
$\mathrm{C} 8-\mathrm{C} 7$ & $1.463(4)$ & $\mathrm{C} 5-\mathrm{H} 5 \mathrm{~A}$ & 0.9300 \\
$\mathrm{C} 6-\mathrm{C} 1$ & $1.379(4)$ & $\mathrm{C} 3-\mathrm{C} 4$ & $1.360(5)$ \\
$\mathrm{C} 6-\mathrm{C} 5$ & $1.381(4)$ & $\mathrm{C} 3-\mathrm{C} 2$ & $1.370(5)$ \\
$\mathrm{C} 6-\mathrm{N} 1$ & $1.405(3)$ & $\mathrm{C} 3-\mathrm{H} 3 \mathrm{~A}$ & 0.9300 \\
$\mathrm{C} 7-\mathrm{N} 1$ & $1.354(3)$ & $\mathrm{C} 2-\mathrm{H} 2 \mathrm{~A}$ & 0.9300 \\
$\mathrm{~N} 2-\mathrm{N} 3$ & $1.308(3)$ & $\mathrm{C} 4-\mathrm{H} 4 \mathrm{~A}$ & 0.9300 \\
$\mathrm{~N} 4-\mathrm{N} 3$ & $1.327(3)$ & $\mathrm{C} 11-\mathrm{H} 11 \mathrm{~A}$ & 0.9600 \\
$\mathrm{~N} 4-\mathrm{C} 9$ & $1.334(3)$ & $\mathrm{C} 11-\mathrm{H} 11 \mathrm{~B}$ & 0.9600
\end{tabular}




\begin{tabular}{|c|c|c|c|}
\hline $\mathrm{N} 4-\mathrm{O} 2$ & $1.382(3)$ & $\mathrm{C} 11-\mathrm{H} 11 \mathrm{C}$ & 0.9600 \\
\hline $\mathrm{C} 9-\mathrm{C} 10$ & $1.488(4)$ & $\mathrm{C} 10-\mathrm{H} 10 \mathrm{~A}$ & 0.9600 \\
\hline $\mathrm{N} 1-\mathrm{H} 1$ & $0.85(3)$ & $\mathrm{C} 10-\mathrm{H} 10 \mathrm{~B}$ & 0.9600 \\
\hline $\mathrm{O} 2-\mathrm{C} 11$ & $1.333(4)$ & $\mathrm{C} 10-\mathrm{H} 10 \mathrm{C}$ & 0.9600 \\
\hline $\mathrm{N} 2-\mathrm{C} 8-\mathrm{C} 9$ & $109.5(2)$ & $\mathrm{C} 4-\mathrm{C} 5-\mathrm{C} 6$ & $120.0(3)$ \\
\hline $\mathrm{N} 2-\mathrm{C} 8-\mathrm{C} 7$ & $122.6(2)$ & $\mathrm{C} 4-\mathrm{C} 5-\mathrm{H} 5 \mathrm{~A}$ & 120.0 \\
\hline $\mathrm{C} 9-\mathrm{C} 8-\mathrm{C} 7$ & $127.8(2)$ & $\mathrm{C} 6-\mathrm{C} 5-\mathrm{H} 5 \mathrm{~A}$ & 120.0 \\
\hline $\mathrm{C} 1-\mathrm{C} 6-\mathrm{C} 5$ & $119.6(3)$ & $\mathrm{C} 4-\mathrm{C} 3-\mathrm{C} 2$ & $120.0(3)$ \\
\hline $\mathrm{C} 1-\mathrm{C} 6-\mathrm{N} 1$ & $118.1(3)$ & $\mathrm{C} 4-\mathrm{C} 3-\mathrm{H} 3 \mathrm{~A}$ & 120.0 \\
\hline $\mathrm{C} 5-\mathrm{C} 6-\mathrm{N} 1$ & $122.3(3)$ & $\mathrm{C} 2-\mathrm{C} 3-\mathrm{H} 3 \mathrm{~A}$ & 120.0 \\
\hline $\mathrm{O} 1-\mathrm{C} 7-\mathrm{N} 1$ & $124.1(3)$ & $\mathrm{C} 3-\mathrm{C} 2-\mathrm{C} 1$ & $120.2(3)$ \\
\hline $\mathrm{O} 1-\mathrm{C} 7-\mathrm{C} 8$ & $120.6(2)$ & $\mathrm{C} 3-\mathrm{C} 2-\mathrm{H} 2 \mathrm{~A}$ & 119.9 \\
\hline $\mathrm{N} 1-\mathrm{C} 7-\mathrm{C} 8$ & $115.3(2)$ & $\mathrm{C} 1-\mathrm{C} 2-\mathrm{H} 2 \mathrm{~A}$ & 119.9 \\
\hline $\mathrm{N} 3-\mathrm{N} 2-\mathrm{C} 8$ & $109.2(2)$ & $\mathrm{C} 5-\mathrm{C} 4-\mathrm{C} 3$ & $120.7(3)$ \\
\hline $\mathrm{N} 3-\mathrm{N} 4-\mathrm{C} 9$ & $115.2(2)$ & $\mathrm{C} 5-\mathrm{C} 4-\mathrm{H} 4 \mathrm{~A}$ & 119.6 \\
\hline $\mathrm{N} 3-\mathrm{N} 4-\mathrm{O} 2$ & $118.1(3)$ & $\mathrm{C} 3-\mathrm{C} 4-\mathrm{H} 4 \mathrm{~A}$ & 119.6 \\
\hline $\mathrm{C} 9-\mathrm{N} 4-\mathrm{O} 2$ & $125.9(2)$ & $\mathrm{O} 2-\mathrm{C} 11-\mathrm{H} 11 \mathrm{~A}$ & 109.5 \\
\hline $\mathrm{N} 4-\mathrm{C} 9-\mathrm{C} 8$ & $101.5(2)$ & $\mathrm{O} 2-\mathrm{C} 11-\mathrm{H} 11 \mathrm{~B}$ & 109.5 \\
\hline $\mathrm{N} 4-\mathrm{C} 9-\mathrm{C} 10$ & $123.7(3)$ & $\mathrm{H} 11 \mathrm{~A}-\mathrm{C} 11-\mathrm{H} 11 \mathrm{~B}$ & 109.5 \\
\hline $\mathrm{C} 8-\mathrm{C} 9-\mathrm{C} 10$ & $134.8(3)$ & $\mathrm{O} 2-\mathrm{C} 11-\mathrm{H} 11 \mathrm{C}$ & 109.5 \\
\hline $\mathrm{C} 7-\mathrm{N} 1-\mathrm{C} 6$ & $126.3(3)$ & $\mathrm{H} 11 \mathrm{~A}-\mathrm{C} 11-\mathrm{H} 11 \mathrm{C}$ & 109.5 \\
\hline $\mathrm{C} 7-\mathrm{N} 1-\mathrm{H} 1$ & $113.4(17)$ & $\mathrm{H} 11 \mathrm{~B}-\mathrm{C} 11-\mathrm{H} 11 \mathrm{C}$ & 109.5 \\
\hline $\mathrm{C} 6-\mathrm{N} 1-\mathrm{H} 1$ & $120.2(17)$ & $\mathrm{C} 9-\mathrm{C} 10-\mathrm{H} 10 \mathrm{~A}$ & 109.5 \\
\hline $\mathrm{N} 2-\mathrm{N} 3-\mathrm{N} 4$ & $104.6(2)$ & C9- $\mathrm{C} 10-\mathrm{H} 10 \mathrm{~B}$ & 109.5 \\
\hline $\mathrm{C} 11-\mathrm{O} 2-\mathrm{N} 4$ & $111.1(3)$ & $\mathrm{H} 10 \mathrm{~A}-\mathrm{C} 10-\mathrm{H} 10 \mathrm{~B}$ & 109.5 \\
\hline $\mathrm{C} 2-\mathrm{C} 1-\mathrm{C} 6$ & $119.6(3)$ & $\mathrm{C} 9-\mathrm{C} 10-\mathrm{H} 10 \mathrm{C}$ & 109.5 \\
\hline $\mathrm{C} 2-\mathrm{C} 1-\mathrm{H} 1 \mathrm{~A}$ & 120.2 & $\mathrm{H} 10 \mathrm{~A}-\mathrm{C} 10-\mathrm{H} 10 \mathrm{C}$ & 109.5 \\
\hline $\mathrm{C} 6-\mathrm{C} 1-\mathrm{H} 1 \mathrm{~A}$ & 120.2 & $\mathrm{H} 10 \mathrm{~B}-\mathrm{C} 10-\mathrm{H} 10 \mathrm{C}$ & 109.5 \\
\hline
\end{tabular}

Hydrogen-bond geometry $\left(\AA,{ }^{\circ}\right)$

\begin{tabular}{lllll}
\hline$D-\mathrm{H} \cdots A$ & $D-\mathrm{H}$ & $\mathrm{H} \cdots A$ & $D \cdots A$ & $D-\mathrm{H} \cdots A$ \\
\hline $\mathrm{N} 1-\mathrm{H} 1 \cdots \mathrm{N} 2$ & $0.86(2)$ & $2.33(3)$ & $2.780(4)$ & $113(2)$ \\
$\mathrm{N} 1-\mathrm{H} 1 \cdots \mathrm{N} 3^{\mathrm{i}}$ & $0.86(2)$ & $2.41(2)$ & $3.184(3)$ & $150(2)$ \\
\hline
\end{tabular}

Symmetry code: (i) $-x+2, y-1 / 2,-z+1 / 2$. 